\title{
Article
}

\section{Acute biomechanical effects of a lightweight, sock-style minimalist footwear design during running; a musculoskeletal simulation and statistical parametric mapping approach}

Sinclair, Jonathan Kenneth, Brooks, Darrell and Stainton, Philip Available at http://clok.uclan.ac.uk/26437/

Sinclair, Jonathan Kenneth ORCID: 0000-0002-2231-3732, Brooks, Darrell ORCID: 0000-0002-4094-5266 and Stainton, Philip (2019) Acute biomechanical effects of a lightweight, sock-style minimalist footwear design during running; a musculoskeletal simulation and statistical parametric mapping approach. Footwear Science, 11 (2). pp. 71-83. ISSN 1942-4280

It is advisable to refer to the publisher's version if you intend to cite from the work. http://dx.doi.org/10.1080/19424280.2019.1593516

For more information about UCLan's research in this area go to

http://www.uclan.ac.uk/researchgroups/ and search for <name of research Group>.

For information about Research generally at UCLan please go to http://www.uclan.ac.uk/research/

All outputs in CLoK are protected by Intellectual Property Rights law, including Copyright law. Copyright, IPR and Moral Rights for the works on this site are retained by the individual authors and/or other copyright owners. Terms and conditions for use of this material are defined in the policies page. 


\section{$\underline{\text { Abstract }}$}

The aim of this study was to examine the effects of existing minimalist footwear, new sockstyle minimalist footwear and conventional running footwear on lower extremity biomechanics, using a musculoskeletal simulation and statistical parametric mapping (SPM) approach. Thirteen male participants ran over an embedded force plate at $4.0 \mathrm{~m} / \mathrm{s}$, in 1 . existing minimalist footwear, 2. new sock-style minimalist footwear and 3. conventional running shoes. Kinematics of the lower extremities were collected using an eight-camera motion analysis system and lower extremity joint loading was also explored using a musculoskeletal simulation approach. Differences between footwear conditions were examined using SPM and one-way repeated measures ANOVA. The strike index indicated that the foot contact position was significantly more anterior in existing minimalist footwear (44.19\%) and new sock-style minimalist footwear (42.33\%) compared to conventional running shoes $(29.00 \%)$. The instantaneous loading rate was also significantly larger in existing minimalist footwear (271.68 BW/s) and new sock-style minimalist footwear (299.26 $\mathrm{BW} / \mathrm{s})$ in relation to conventional running shoes $(122.48 \mathrm{BW} / \mathrm{s})$. In addition, during the late stance phase compressive hip joint loading was significantly larger in both minimalist footwear. Similarly, Achilles tendon loading was statistically greater in both minimalist footwear compared to the conventional running shoe during the early and middle aspects of the stance phase. The observations from this analysis show that minimalist footwear may 
place non-habituated runners at greater risk from the mechanical factors linked to the

\section{Introduction}

Running is one of the most popular aerobic exercise modalities, and there is an overwhelming

body of evidence that it mediates a plethora of physiological and psychological benefits (Lee

et al., 2014). However, running is also associated with an extremely high susceptibility to

chronic pathologies; with up to $80 \%$ of runners experiencing an injury each year (Van Gent

32 et al., 2007). Chronic injuries are a key barrier to training compliance (Hespanhol et al.,

2016), and result in a significant economic burden due to healthcare operation and absence

from work (Junior et al., 2017).

As the primary interface between foot and ground, running shoes are proposed as a mechanism by which the rate of chronic injuries can be moderated (Shorten, 2000). However, since the introduction of the conventional running shoe in the 1970 's, the rate and location of chronic running injuries has remained unchanged (Davis, 2014). This has led to the

supposition that reverting to running in minimalist footwear that lacks the mechanical

properties associated with the conventional running shoe, may be associated with a reduced 
46 Several studies have explored biomechanical differences between minimalist and

47 conventional running shoes. These analyses have typically examined spatiotemporal

48 characteristics, lower limb kinematics and loading rates. Sinclair et al., (2013a) and Sinclair

49 et al., (2016) showed that minimalist footwear caused runners to run with a more

50 plantarflexed ankle at initial contact, increased peak tibial internal rotation and an increased

51 vertical loading rate in comparison to conventional running shoes. Squadrone et al., (2009)

52 similarly showed that running in minimalist footwear increased the ankle plantarflexion angle

53 at footstrike but also reduced stride length and the impact peak of the vertical ground reaction

54 force (GRF). Squadrone et al., (2015) investigated the effects of different minimalist

55 footwear conditions via the strike index. Their findings showed that minimalist footwear

56 mediated a midfoot strike pattern, with alterations being most pronounced in footwear with

57 the least midsole cushioning. Sinclair et al., (2018) showed that the strike index did not

58 change between different minimalist footwear models and conventional running shoes, but

59 did find that effective mass was significantly larger in minimalist footwear with alterations

60 again being more evident in models with the least midsole cushioning.

62 Previous work has also examined the effects of minimalist footwear on the loads experienced 63 by the lower extremities joint during running. Sinclair, (2014) and Sinclair et al., (2016) showed that peak patellofemoral stress was significantly reduced in minimalist footwear, but peak Achilles tendon loads were significantly increased. Similarly, Bonacci et al., (2018) showed that peak patellofemoral stress was significantly lower in minimalist footwear. In addition, Sinclair, (2016) showed that peak tibiofemoral loading did not differ significantly between minimalist and conventional footwear during running. Furthermore, Sinclair et al., 69 (2015) and Sinclair et al., (2016) taking into account the effect of changing stride length 
71 significantly reduced but Achilles tendon impulse per mile was significantly greater in

72 minimalist footwear, with differences being more evident in minimalist footwear with the

73 least midsole cushioning. Recently, a new lightweight, sock-style minimalist footwear design

74 has been commercially released, which represents an extremely lightweight sock style upper

75 with a strong abrasion resistant sole. There are however, no published scientific

76 investigations concerning this new footwear, indicating that examination of running

77 biomechanics whilst wearing these shoes is warranted.

79 Previous analyses concerning the biomechanical differences between minimalist and

conventional footwear, have utilized mathematical modelling approaches driven by joint

torques to explore the loads experienced by the musculoskeletal system. However, joint

torques are global indices of joint loading, and therefore not representative of localized joint

loading (Herzog et al., 2003a). Herzog et al., (2003b) identified importantly that the muscles are the primary contributors to lower extremity joint loading. Due to the difficulties

associated with calculating muscle kinetics, the role of the muscles in controlling joint

biomechanics during running has received little attention within biomechanical literature.

87 Over the past decade however, significant advances have been made in improving 88 musculoskeletal models; leading to the development of open access and bespoke software.

89 Allowing skeletal muscle forces to be simulated during movement, and utilized as inputs to

90 calculate lower extremity joint reaction forces (Delp et al., 2007). Such approaches have not

91 yet been utilized to explore biomechanical differences between minimalist and conventional

92 running shoes. 
94 To date biomechanical differences between minimalist and conventional footwear have been

95

96

97

\section{$114 \quad$ Methods}

Participants

To summarize, there is currently no scientific research concerning the aforementioned sockstyle minimalist footwear, nor is there any investigation which has collectively explored the effects of minimalist and conventional running shoes using both musculoskeletal simulation and SPM. Therefore, the aim of the current investigation was to examine the effects of existing/ sock-style minimalist footwear and conventional running shoes on lower extremity biomechanics using a musculoskeletal simulation and SPM based approach. A study of this nature may provide further insight into the biomechanical differences between minimalist and traditional running shoes; particularly with regards to runners' predisposition to chronic running injuries.

Thirteen male runners volunteered to take part in this study. This sample size is commensurate with previous analyses concerning the biomechanics of running in minimalist 


6

footwear (Sinclair et al., 2013a; Sinclair et al., 2015). The mean characteristics of the participants were: age $27.31 \pm 3.50$ years, height $1.73 \pm 0.04 \mathrm{~m}$ and body mass $72.23 \pm 5.66$ $\mathrm{kg}$. The procedure utilized for this investigation was approved by the University of Central Lancashire, Science, Technology, Engineering and Mathematics, ethical committee. All runners were free from musculoskeletal pathology at the time of data collection. Participants provided written informed consent in accordance with the principles outlined in the Declaration of Helsinki.

\section{Footwear}

The footwear used during this study consisted of New Balance, 1260 v2 (New Balance, Boston, Massachusetts, United States; henceforth termed Shoe A), Vibram Five-Fingers, ELX (Vibram, Albizzate, Italy; henceforth termed Shoe B) and Skinners, Athleisure (Skinners Technologies, Cyrilska, Czech Republic; henceforth termed Shoe C) (Figure 1).

Shoe A had an average mass of $0.285 \mathrm{~kg}$, heel thickness of $25 \mathrm{~mm}$ and a heel drop of $14 \mathrm{~mm}$. Shoe B had an average mass of $0.167 \mathrm{~kg}$, heel thickness of $7 \mathrm{~mm}$ and a heel drop of $0 \mathrm{~mm}$. Finally, Shoe C had an average mass of $0.08 \mathrm{~kg}$, heel thickness of $6 \mathrm{~mm}$ and a heel drop of 0 $\mathrm{mm}$. The footwear were also scored using the minimalist index described by Esculier et al., (2015), and Shoe A received a score of 20, Shoe B a score of 92 and Shoe C a score of 100. 
140 Participants ran at $4.0 \mathrm{~m} / \mathrm{s}( \pm 5 \%)$, striking an embedded piezoelectric force platform (Kistler 141 Instruments Ltd., Winterthur, Switzerland) with their right foot. Running velocity was 142 monitored using infrared timing gates (Newtest, Oy Koulukatu, Finland). The stance phase was delineated as the duration over which $20 \mathrm{~N}$ or greater of vertical GRF was applied to the force platform. Runners completed a minimum of five successful trials in each footwear condition. As each footwear were novel to all participants, a period of 5 minutes for accommodation was allowed. This involved running through the testing area without concern participants ran in each footwear condition was counterbalanced. Kinematic and GRF data were synchronously collected. Kinematic data were captured at $250 \mathrm{~Hz}$ via an eight-camera motion analysis system (Qualisys Medical AB, Goteburg, Sweden). Dynamic calibration of the motion capture system was performed before each data collection session.

Lower extremity segments were modelled in 6 degrees of freedom using the calibrated anatomical systems technique (Cappozzo et al., 1995). To define the anatomical frames of the thorax, pelvis, thighs, shanks and feet retroreflective markers were placed at the C7, T12 and xiphoid process landmarks and also positioned bilaterally onto the acromion process, iliac crest, anterior superior iliac spine (ASIS), posterior super iliac spine (PSIS), medial and lateral malleoli, medial and lateral femoral epicondyles, greater trochanter, calcaneus, first metatarsal and fifth metatarsal. Carbon-fibre tracking clusters comprising of four non-linear retroreflective markers were positioned onto the thigh and shank segments. In addition to these, the foot segments were tracked via the calcaneus, first metatarsal and fifth metatarsal, the pelvic segment was tracked using the PSIS and ASIS markers and the thorax segment was tracked using the T12, C7 and xiphoid markers. Static calibration trials (not normalized to 
static trial posture) were obtained in each footwear allowing for the anatomical markers to be referenced in relation to the tracking markers/ clusters.

Processing

Dynamic trials were digitized using Qualisys Track Manager (Qualisys Medical AB, Goteburg, Sweden) in order to identify anatomical and tracking markers then exported as C3D files to Visual 3D (C-Motion, Germantown, MD, USA). All data were linearly normalized to $100 \%$ of the stance phase. GRF data and marker trajectories were smoothed with cut-off frequencies of $50 \mathrm{~Hz}$ at $12 \mathrm{~Hz}$ respectively, using a low-pass Butterworth 4th order zero lag filter. All force parameters throughout were normalized by dividing by bodyweight (BW).

In accordance with the protocol of Addison \& Lieberman, (2015), an impulse-momentum modelling approach was utilized to calculate effective mass (\% BW), which was quantified in accordance with the below equation:

$$
\text { Effective mass }=\text { vertical GRF integral } /(\Delta \text { foot vertical velocity }+g * \Delta \text { time })
$$

The impact peak was defined in Shoe A as the first peak in vertical GRF. In Shoes B and C where no impact peak was present, according to the protocols of Lieberman et al., (2010) and Sinclair et al., (2018) we defined the position of the impact peak at the same relative position as in Shoe A, which was shown to be $11.96 \%$ of the stance phase. The time (ms) to impact peak ( $\triangle$ time) was quantified as the duration from footstrike to impact peak. The vertical GRF 
integral $(\mathrm{BW} \cdot \mathrm{ms})$ during the period of the impact peak was calculated using a trapezoidal function. The change in foot vertical velocity ( $\triangle$ foot vertical velocity) was determined as the instantaneous vertical foot velocity averaged across the 10 frames prior to the impact peak (Sinclair et al., (2018). The velocity of the foot was quantified using the centre of mass of the foot segment in the vertical direction, within Visual 3D (Sinclair et al., 2018).

\section{Instantaneous loading rate (BW/s) was also was also extracted by obtaining the peak increase} in vertical GRF between adjacent data points. Finally, the strike index was calculated as the position of the centre of pressure location at footstrike, relative to the total length of the foot (Squadrone et al., 2015). A strike index of 0-33\% denotes a rearfoot, 34-67\% a midfoot and 68-100\% a forefoot strike pattern.

Following this, data during the stance phase were exported from Visual 3D into OpenSim 3.3 software (Simtk.org). A validated musculoskeletal model with 12 segments, 19 degrees of freedom and 92 musculotendon actuators (Lerner et al., 2015) was used to estimate lower extremity joint forces. The model was scaled to account for the anthropometrics of each athlete. As muscle forces are the main determinant of joint compressive forces (Herzog et al., 2003), muscle kinetics were quantified using static optimization in accordance with Steele et al., (2012). Compressive patellofemoral, medial/ lateral tibiofemoral and hip joint forces were calculated via the joint reaction analyses function using the muscle forces generated from the static optimization process as inputs. Finally, Achilles tendon forces were estimated in accordance with the protocol of Almonroeder et al., (2013), by summing the muscle forces of the medial gastrocnemius, lateral, gastrocnemius, and soleus muscles. 
during the stance phase, using a trapezoidal function. In addition to this, we also estimated

the total impulse per kilometre $(\mathrm{BW} \cdot \mathrm{km})$ by multiplying these parameters by the number of

steps required to run a kilometre. The number of steps required to complete one kilometre

217 was quantified using the step length $(\mathrm{m})$, which was determined by taking the difference in

Compressive joint forces (hip, patellofemoral, medial tibiofemoral and lateral tibiofemoral),

Achilles tendon loading and three-dimensional kinematics during the entire stance phase

were temporally normalized using linear interpolation to 101 data points. Differences across

the entire stance phase were examined using 1-dimensional SPM with MATLAB 2017a

(MATLAB, MathWorks, Natick, USA), in accordance with Pataky et al., (2016), using the

source code available at http://www.spm1d.org/. In agreement with Pataky et al., (Pataky et

al., 2013), SPM was implemented in a hierarchical manner, analogous to one-way repeated

measures ANOVA (SPM F) with post-hoc paired t-tests (SPM t). Therefore, the entire data

set was examined first, and if a statistical main effect was reached, then post-hoc tests were

conducted on each component separately.

232 For discrete parameters that could not be examined using SPM (hip impulse per km, lateral 
and standard deviations were calculated for each outcome measurement for all footwear

conditions. Differences in discrete biomechanical parameters between footwear were

examined using one-way repeated measures ANOVAs, Effect sizes were calculated using

partial eta ${ }^{2}\left(\mathrm{p \eta}^{2}\right)$. In the event of a significant main effect, post-hoc pairwise comparisons

were conducted on all significant main effects, using a Bonferroni adjustment. Discrete

statistical actions were conducted using SPSS v24.0 (SPSS Inc., Chicago, USA). Statistical

significance for main effects was accepted at the $\mathrm{P} \leq 0.05$ level (Sinclair et al., 2013b).

\section{$\underline{\text { Results }}$}

A main effect was revealed for the instantaneous loading rate $\left(\mathrm{P}<0.001, \mathrm{p} \eta^{2}=0.75\right)$. Post-hoc analyses showed that instantaneous loading rate was significantly larger in Shoe $B(P<0.001)$ and Shoe C $(\mathrm{P}<0.001)$, compared to Shoe A (Table 1).

A main effect was shown for strike index $\left(\mathrm{P}=0.033, \mathrm{p}^{2}=0.27\right)$. Post-hoc analyses showed that strike index was significantly larger in Shoe B $(\mathrm{P}=0.008)$ and Shoe $\mathrm{C}(\mathrm{P}=0.006)$, compared to Shoe A (Table 1). 
257 A main effect was evident for effective mass $\left(\mathrm{P}=0.005, \mathrm{p} \eta^{2}=0.38\right)$. Post-hoc analyses 258 showed that effective mass was significantly larger in Shoes A $(\mathrm{P}=0.01)$ and $\mathrm{C}(\mathrm{P}=0.04)$, 259 compared to Shoe B (Table 1). Finally, a main effect was shown for step length $(\mathrm{P}=0.012$, $260 \mathrm{p \eta ^{2 }}=0.33$ ). Post-hoc analyses showed that step length was significantly larger in Shoe A 261 compared to Shoe C $(\mathrm{P}=0.005)$ (Table 1).

262

Joint loading per kilometre

At the hip joint a main effect was found for peak hip impulse per kilometre $\left(\mathrm{P}=0.018, \mathrm{p} \eta^{2}=\right.$ 265

0.31). Post-hoc analysis showed that hip impulse per kilometre was significantly larger in

Shoe C compared to shoe A $(\mathrm{P}=0.004)$ (Table 1$)$.

There was also a main effect for patellofemoral impulse per kilometre $\left(\mathrm{P}=0.029, \mathrm{p} \eta^{2}=0.28\right)$.

Post-hoc analysis showed that patellofemoral impulse per kilometre was significantly larger in Shoe C compared to shoe B $(\mathrm{P}=0.02)$ (Table 1).

0.58). Post-hoc analyses showed that Achilles tendon impulse per kilometre was significantly larger in Shoes B $(\mathrm{P}=0.001)$ and $\mathrm{C}(\mathrm{P}=0.002)$ compared to shoe A (Table 1). 
282 At the hip joint, there was a significant main effect (Figure 4a). Post-hoc analyses showed that Shoe A was associated with lower compressive hip force than Shoes B and C, from 82$88 \%$ of the stance phase (Figure $4 \mathrm{bc}$ ).

At the patellofemoral joint, there was a significant main effect (Figure 4d). Post-hoc analyses showed that Shoe A was associated with lower patellofemoral force than Shoe B from 81$90 \%$ of the stance phase (Figure $4 \mathrm{e}$ ).

At the medial aspect of the tibiofemoral joint, there was also a main effect (Figure 4f). Posthoc analyses showed that Shoe A was associated with lower compressive force than Shoe B from $5-10 \%$ and $80-92 \%$ of the stance phase (Figure $4 \mathrm{~g}$ ). In addition, Shoe A was associated with lower compressive loading than Shoe C from 5-10\% of the stance phase yet greater loading from 4-9\% of the stance phase (Figure 4h).

296 At the lateral aspect of the tibiofemoral joint, there was also a main effect (Figure 5a). Posthoc analyses showed that Shoe A was associated with lower compressive force than Shoe B $82-89 \%$ of the stance phase (Figure $5 \mathrm{~b}$ ). In addition, Shoe A was associated with lower compressive force than Shoe C, between 0-3\% of the stance phase (Figure 5c). 
301 At the Achilles tendon, there was a main effect (Figure 5d). Post-hoc analyses showed that

302 Shoe A was associated with lower tendon loading than Shoe B, between 7-12\%, 17-55\% and $30382-92 \%$ of the stance phase (Figure 5e). In addition, Shoe A was associated with lower 304 tendon loading compared to Shoe C, from $0-3 \%, 20-25 \%$ and $35-50 \%$ of the stance phase 305 (Figure 5f).

Statistical parametric mapping - three-dimensional kinematics

308

309

\section{Discussion}

For tibial internal rotation, there was a main effect (Figure 5g). Post-hoc analyses showed that Shoe A was associated with increased tibial internal rotation than Shoe B, between $0-5 \%$ and $90-100 \%$ of the stance phase (Figure $5 \mathrm{~h}$ ).

At the ankle in the sagittal plane, there was a main effect (Figure 6a). Post-hoc analyses showed that Shoe A was significantly more dorsiflexed than Shoe B, from 0-3\% of the stance phase (Figure 6b). In addition, it was revealed that Shoe A was significantly more dorsiflexed than Shoe C, from 0-8\% of the stance phase (Figure 6c).

The current investigation aimed to examine the effects of existing/ sock-style minimalist footwear and conventional running shoes on lower extremity biomechanics using a musculoskeletal simulation and SPM based approach. To the authors knowledge this is the first investigation to comparatively examine these footwear and to explore the biomechanics of running in conventional and minimalist footwear using musculoskeletal simulation and 
The kinematic analysis using SPM showed that the ankle was in a significantly more plantarflexed position during the early stance phase in Shoes B and C in comparison to Shoe A. This observation is reinforced by the discrete point analysis of the strike index, which showed that the contact position was significantly more anterior in Shoes B and C, and a midfoot strike pattern was adopted when wearing these footwear. This finding concurs with the observations of Sinclair et al., (2013a) and Sinclair et al., (2016) who each showed an altered foot position when wearing minimalist footwear. It is proposed that this relates to the absence of cushioning in Shoes B and C, causing runners to adopt a flatter foot position in order to compensate for the lack of midsole interface in an attempt to attenuate the load experienced by the lower extremities (Lieberman et al., 2010).

The findings from the current investigation also showed that the instantaneous loading rate was significantly larger and the effective mass was significantly lower in Shoes B and C compared to Shoe A. This observation agrees with those of Sinclair et al., (2013a) and Sinclair et al., (2016) but opposes those of Squadrone \& Gallozzi, (2009) and Sinclair et al., (2018). Transient loading is governed by the rate at which the momentum of the foot changes, therefore midsole material at the foot-ground interface strongly influences the magnitude of transient forces during running (Whittle, 1999). Importantly, Addison \& Liebermann, (2015) found that the loading rate and effective mass were inversely associated during running. Therefore, the aforementioned observation in relation to the loading rate is supported by the effective mass observations, which was shown to be reduced in Shoes B and

C compared to Shoe A. Given the proposed association between the instantaneous rate of 
348 (Milner et al., 2006), and indicates that Shoes B and C may place runners at increased risk 349 from impact related injuries compared to Shoe A.

351 At the hip joint, the current investigation showed using SPM, that Shoe A significantly reduced compressive hip joint loading during the early and late aspects of the stance phase compared to Shoes B and C. This observation is supported through the discrete point analysis, which showed that compressive joint forces experienced per kilometre were statistically greater in Shoe C compared to shoe A. As the current investigation represents the first investigation to compare hip joint loading when running in minimalist and conventional footwear using musculoskeletal simulation, comparisons in relation to previous analyses are not possible. Nonetheless, the results are partially supported by those of Rooney \& Derrick, (2013) and Sinclair, (2018) who showed that modifying the foot position significantly enhanced compressive hip joint loading during running. As the aetiology of hip joint pathologies are strongly influenced by compressive hip joint loading (Johnson \& Hunter, 2014), the current investigation indicates that Shoes B and C may increase runners' susceptibility to chronic hip pathologies.

A further important observation from the current analysis is that patellofemoral loading contrasted using SPM was statistically larger in Shoe B compared to Shoe A during late stance. The discrete analysis differed from this, showing that patellofemoral force per current investigation oppose those of Sinclair, (2014), Sinclair et al., (2016) and Bonacci et 

indicates firstly that running in minimalist footwear may not necessarily attenuate the magnitude of patellofemoral loading linked to the aetiology of patellofemoral disorders during running, in relation to conventional running shoes. Furthermore, the current study revealed that patellofemoral was statistically larger in Shoe $\mathrm{C}$ compared to shoe $\mathrm{B}$, indicating that despite their relatively similar design characteristics (Esculier et al., 2015); Shoe C may place runners at increased risk from patellofemoral chronic injuries.

At the medial and lateral tibiofemoral joint compartments, compressive loading was significantly greater in Shoes B and C in relation to Shoe A, during the early and late aspects of the stance phase. This observation opposes those of Sinclair, (2016) but is supported

may be clinically meaningful, as increased compressive loading at both aspects of the tibiofemoral joint, is recognised as the primary risk factor in relation to the aetiology and progression of osteoarthritic symptoms (Dabiri \& Li, 2013). Therefore, the current study shows that indicates that running in minimalist footwear may increase runners predisposition to the risk factors linked to the initiation of tibiofemoral osteoarthritis. The findings from the current investigation also revealed using SPM that Achilles tendon loading was statistically larger during the mid and late aspects of the stance phase in Shoes B 
and C compared to Shoe A. In addition, the discrete point analysis of tendon loading per

397 kilometre similarly indicated that Shoes B and C were associated with statistically larger tendon loading magnitudes. This observation concurs with those of Sinclair, (2014) and Sinclair et al., (2015) who similarly showed that peak Achilles tendon force and tendon impulse per mile were greater when running in minimalist footwear in comparison to conventional running shoes. The aetiology of Achilles tendinopathy is associated with excessive and repeated tendinous loading, during cyclic activities such as running (Magnusson et al., 2010). Excessive tendon loading without sufficient caseation of running activities between training sessions, mediates collagen and extracellular matrix synthesis and degradation of the tendon (Magnusson et al., 2010). As such, the current investigation shows that running in minimalist footwear may place runners at increased risk from the biomechanical parameters linked to Achilles tendinopathy, in comparison to conventional running shoes.

A potential limitation that should be acknowledged in regards to the current investigation is of course that only runners who habitually ran in conventional running shoes were examined.

412 The findings from previous analyses concerning the biomechanics of minimalist footwear 413 and conventional running shoes have drawn opposing interpretations, frequently on the basis of the running experience of the participants in minimalist footwear (Sinclair et al., 2013a; Squadrone \& Gallozzi, 2009). It can therefore be ventured that the findings from the current investigation may have been different, had the participants been habitual minimalist footwear users. As such, future analyses using musculoskeletal simulation and SPM investigating the 418 biomechanics of running in habitual minimalist footwear is recommended, allowing more 419 decisive assertions in regards to the aetiology of chronic pathologies to be drawn. 
421 In conclusion, though the biomechanics of running in minimalist and conventional running

422 footwear have received widespread research attention, there has not yet been a quantitative

423 comparison of lower extremity biomechanics in minimalist and conventional running shoes

424 using a musculoskeletal simulation and SPM based approach. This study revealed that the

425 instantaneous load rate, hip, tibiofemoral and Achilles tendon force parameters were

426 statistically larger when running in Shoes B and C compared to Shoe A. Therefore, the

427 observations from this analysis show that minimalist footwear may place non-habituated

428 runners at greater risk from the mechanical factors linked to the aetiology of chronic lower

429 limb running related injuries.

\section{$\underline{\text { References }}$}

1. Addison, B.J., Lieberman, D.E. (2015). Tradeoffs between impact loading rate,

footwear of varying stiffness. Journal of Biomechanics, 48, 1318-1324.

2. Almonroeder, T., Willson, J.D., Kernozek, T.W. (2013). The effect of foot strike pattern on Achilles tendon load during running. Annals of Biomedical Engineering,

\section{1, 1758-1766.}

3. Bonacci, J., Hall, M., Fox, A., Saunders, N., Shipsides, T., Vicenzino, B. (2018). The influence of cadence and shoes on patellofemoral joint kinetics in runners with patellofemoral pain. Journal of Science and Medicine in Sport, 21, 574-578.

4. Cappozzo, A., Catani, F., Leardini, A., Benedeti, M.G., Della, CU. (1995). Position and orientation in space of bones during movement: Anatomical frame definition and determination. Clinical Biomechanics, 10, 171-178. 
5. Dabiri, Y., Li, L.P. (2013). Altered knee joint mechanics in simple compression associated with early cartilage degeneration. Computational and Mathematical Methods in Medicine, 11, 1-12.

6. Davis, I.S. (2014). The re-emergence of the minimal running shoe. Journal of Orthopaedic \& Sports Physical Therapy, 44, 775-784.

7. Delp, S.L., Anderson, F.C., Arnold, A.S., Loan, P., Habib, A., John, C.T., Thelen, D.G. (2007). OpenSim: open-source software to create and analyze dynamic simulations of movement. IEEE Transactions in Biomedical Engineering, 54, 19401950.

8. Esculier, J.F., Dubois, B., Dionne, C.E., Leblond, J., Roy, J.S. (2015). A consensus definition and rating scale for minimalist shoes. Journal of Foot and Ankle Research, $8,1-9$.

9. Herzog W, Longino D, Clark A (2003a). The role of muscles in joint adaptation and degeneration. Langenbecks Archives of Surgery, 388, 305-315.

10. Herzog, W., Clark, A., Wu, J. (2003b). Resultant and local loading in models of joint disease. Arthritis Care Research, 49, 239-247.

11. Hespanhol, L.C., Van Mechelen, W., Postuma, E., Verhagen, E. (2016). Health and economic burden of running-related injuries in runners training for an event: A prospective cohort study. Scandinavian Journal of Medicine \& Science in Sports, 26, 1091-1099.

12. Johnson, V.L., Hunter, D.J. (2014). The epidemiology of osteoarthritis. Best Practice \& Research: Clinical Rheumatology, 28, 5-15.

13. Junior, L.C.H., Van Mechelen, W., Verhagen, E. (2017). Health and economic burden of running-related injuries in Dutch trailrunners: a prospective cohort study. Sports Medicine, 47, 367-377. 

time running reduces all-cause and cardiovascular mortality risk. Journal of the American College of Cardiology, 64, 472-481.

15. Lerner, Z.F., DeMers, M.S., Delp, S.L., Browning, R.C. (2015). How tibiofemoral contact forces. Journal of Biomechanics, 48, 644-650.

16. Lieberman, D.E., Venkadesan, M., Werbel, W.A., Daoud, A.I., D'Andrea, S., Davis, I.S., Mang'eni, R.O., Pitsiladis, Y. (2010). Foot strike patterns and collision forces in habitually barefoot versus shod runners. Nature, 463, 531-535.

17. Magnusson, S.P., Langberg, H., Kjaer, M. (2010). The pathogenesis of tendinopathy: balancing the response to loading. Nature Reviews Rheumatology, 6, 262-268.

18. Milner, C.E., Ferber, R., Pollard, C.D., Hamill, J., Davis, I.S. (2006). Biomechanical factors associated with tibial stress fracture in female runners. Medicine \& Science in Sports \& Exercise, 38, 323-328.

19. Pataky, T.C., Robinson, M.A., Vanrenterghem, J. (2013). Vector field statistical analysis of kinematic and force trajectories. Journal of Biomechanics, 46, 2394-2401.

20. Pataky, T.C., Robinson, M.A., Vanrenterghem, J. (2016). Region-of-interest analyses of one-dimensional biomechanical trajectories: bridging $0 \mathrm{D}$ and $1 \mathrm{D}$ theory, augmenting statistical power. Peer J, 4, 2652-2664

21. Rooney, B.D., Derrick, T.R. (2013). Joint contact loading in forefoot and rearfoot strike patterns during running. Journal of Biomechanics, 46, 2201-2206.

22. Shorten, MA. (2000). Running shoe design: protection and performance, in Marathon Medicine (Ed. D. Tunstall Pedoe) London, Royal Society of Medicine. 2000; 159 169. 

influence of barefoot and barefoot-inspired footwear on the kinetics and kinematics of running in comparison to conventional running shoes. Footwear Science, 5, 45-53.

24. Sinclair, J., Taylor, P.J., Hobbs, S.J. (2013b). Alpha level adjustments for multiple dependent variable analyses and their applicability-a review. International Journal of Sports Science \& Engineering, 7, 17-20.

25. Sinclair, J. (2014). Effects of barefoot and barefoot inspired footwear on knee and ankle loading during running. Clinical biomechanics, 29, 395-399.

26. Sinclair, J., Richards, J., Shore, H. (2015). Effects of minimalist and maximalist footwear on Achilles tendon load in recreational runners. Comparative Exercise Physiology, 11, 239-244.

27. Sinclair, J. (2016). Minimalist footwear does not affect tiobiofemoral stress loading during the stance phase in rearfoot strikers who use conventional footwear. Comparative Exercise Physiology, 12, 99-103.

28. Sinclair, J., Richards, J., Selfe, J., Fau-Goodwin, J., Shore, H. (2016). The influence of minimalist and maximalist footwear on patellofemoral kinetics during running. Journal of Applied Biomechanics, 32, 359-364.

29. Sinclair, J. (2018). Effects of barefoot and shod running on lower extremity joint loading, a musculoskeletal simulation study. Sport Sciences for Health, (In press).

30. Sinclair, J., Stainton, P., Hobbs, S.J. (2018). Effects of barefoot and minimally shod footwear on effective mass-implications for transient musculoskeletal loading. Kinesiology: Kinesiology, 50, 90-92.

31. Squadrone, R., Gallozzi, C. (2009). Biomechanical and physiological comparison of barefoot and two shod conditions in experienced barefoot runners. Journal of Sports Medicine \& Physical Fitness, 49, 6-13. 
32. Squadrone, R., Rodano, R., Hamill, J., Preatoni, E. (2015). Acute effect of different minimalist shoes on foot strike pattern and kinematics in rearfoot strikers during running. Journal of Sports Sciences, 33, 1196-1204.

33. Steele, K.M., DeMers, M.S., Schwartz, M.H., Delp, S.L. (2012). Compressive tibiofemoral force during crouch gait. Gait \& Posture, 35, 556-560.

34. van Gent, B.R., Siem, D.D., van Middelkoop, M., van Os, T.A., Bierma-Zeinstra, S.S., Koes, B.B. (2007). Incidence and determinants of lower extremity running injuries in long distance runners: a systematic review. British Journal of Sports Medicine, 41, 469-480.

35. Warmenhoven, J., Harrison, A., Robinson, M. A., Vanrenterghem, J., Bargary, N., Smith, R., \& Pataky, T. (2018). A force profile analysis comparison between functional data analysis, statistical parametric mapping and statistical non-parametric mapping in on-water single sculling. Journal of Science and Medicine in Sport, 21, 1100-1105.

36. Whittle, M.W. (1999). The generation and attenuation of transient forces beneath the foot; a review. Gait \& Posture, 10, 264-275.

\section{$\underline{\text { List of figures }}$}

Figure 1: Experimental footwear $(\mathrm{A}=$ New Balance/ Shoe $\mathrm{A}, \mathrm{B}=$ Vibram Five-Fingers/ Shoe B and $\mathrm{C}=$ Skinners/ Shoe C).

Figure 2: Hip, knee and ankle kinematics in the a. sagittal, b. coronal and c. transverse planes as a function of footwear $($ black $=$ Shoe $\mathrm{A}$, dash $=$ Shoe B and grey $=$ Shoe $\mathrm{C}),(\mathrm{FL}=$ flexion, $\mathrm{AD}=$ adduction, $\mathrm{IN}=$ inversion, $\mathrm{INT}=$ internal, $\mathrm{EXT}=$ external $).$ 
541 Figure 3: Lower extremity joint loading as a function of footwear $($ black $=$ Shoe $\mathrm{A}$, dash $=$ 542 Shoe $\mathrm{B}$ and grey $=$ Shoe $\mathrm{C}),(\mathrm{a} .=$ hip, $\mathrm{b} .=$ patellofemoral, $\mathrm{c} .=$ medial tibiofemoral, $\mathrm{d} .=$ 543 lateral tibiofemoral and e. Achilles tendon).

544 Figure 4: Statistical parametric mapping results in relation to lower extremity joint loading (a. 545 hip force main effect, b. hip force Shoe A vs. Shoe B, c. hip force Shoe A vs. Shoe C, d. 546 patellofemoral force main effect, e. patellofemoral force Shoe A vs Shoe B, f. medial 547 tibiofemoral force main effect, g. medial tibiofemoral force Shoe A vs Shoe B, h. medial 548 tibiofemoral force Shoe B vs Shoe C).

549 Figure 5: Statistical parametric mapping results in relation to lower extremity joint loading 550 and joint angles (a. lateral tibiofemoral force main effect, b. lateral tibiofemoral force Shoe A 551 vs. Shoe B, c. lateral tibiofemoral force Shoe A vs. Shoe C, d. Achilles tendon force main 552 effect, e Achilles tendon force Shoe A vs Shoe B, f. Achilles tendon force Shoe A vs. Shoe C, 553 g. tibial internal rotation main effect, h. tibial internal rotation Shoe A vs. Shoe B).

554 Figure 6: Statistical parametric mapping results in relation to sagittal ankle joint angles (a. = 555 ankle angle main effect, b. ankle angle Shoe A vs Shoe B, c. Shoe A vs Shoe C). 